\title{
The Sterol Trafficking Pathway in Arabidopsis thaliana
}

\author{
Krishna Kumar ${ }^{1}$, Holly C. Gibbs ${ }^{2,3}$, Alvin T. Yeh ${ }^{2}$ and Lawrence R. Griffing ${ }^{1,4 *}$ \\ ${ }^{1}$ Molecular and Environmental Plant Sciences Interdisciplinary Program, Texas A\&M University, College Station, TX, \\ United States, ${ }^{2}$ Department of Biomedical Engineering, Texas A\&M University, College Station, TX, United States, \\ ${ }^{3}$ Microscopy and Imaging Center, Texas A\&M University, College Station, TX, United States, ${ }^{4}$ Department of Biology, Texas \\ A\&M University, College Station, TX, United States
}

\section{OPEN ACCESS}

Edited by:

Patrick J. Hussey,

Durham University, United Kingdom

Reviewed by:

Alessandro Vitale,

National Research Council (CNR), Italy

Rosemary White,

Commonwealth Scientific

and Industrial Research Organisation

(CSIRO), Australia

*Correspondence:

Lawrence R. Griffing

griffing@tamu.edu

Specialty section:

This article was submitted to

Plant Cell Biology,

a section of the journal

Frontiers in Plant Science

Received: 12 October 2020

Accepted: 12 April 2021

Published: 26 May 2021

Citation:

Kumar K, Gibbs HC, Yeh AT and

Griffing LR (2021) The Sterol

Trafficking Pathway in Arabidopsis

thaliana. Front. Plant Sci. 12:616631.

doi: 10.3389/fpls.2021.616631
In plants, the trafficking mechanisms by which sterols move through the plant and into target cells are unknown. Earlier studies identified endosomes as primary candidates for internalization of sterols in plants, but these results have come into question. Here, we show that in elongating root cells, the internalization of sterol occurs primarily by a non-endocytic mechanism. Added fluorescent sterols [dehydroergosterol (DHE) and BODIPY-cholesterol (BCh)] do not initially label endosomes identified by fluorescent protein markers or by internalized FM4-64. Instead, the nuclear envelope, an organelle not associated with the endocytic pathway but part of the endoplasmic reticulum $(E R)$, becomes labeled. This result is supported by experiments with the inducible overexpression of auxilin-2-like protein (AUX2 line), which blocks most endocytosis upon induction. Internalization and nuclear envelope labeling still occur in induced AUX2 cells. Longer-term incubation labels the oil body, a site involved in sterol storage. Although the first site of localization, the nuclear envelope, is part of the ER, other domains of the ER do not accumulate the label. The trafficking pathway differs from vesicular endocytosis and points toward a different pathway of sterol transport possibly involving other mechanisms, such as ER-plasma membrane contact sites and cytoplasmic transport.

Keywords: endocytosis, sterol uptake, sterol transport, ER transport, nuclear envelope, oil body

\section{INTRODUCTION}

The movement of sterols, in particular cholesterol, in the plant is of interest for a variety of reasons. First, although the claim of being "cholesterol-free" is made in the United States for many plantbased food products (based on Food and Drug Administration guidelines), plants do indeed have cholesterol (Behrman and Gopalan, 2005), and the genetics of the pathway for the synthesis of cholesterol have recently been outlined (Sonawane et al., 2016). Although cholesterol is about 6\% of the sterol content in Arabidopsis, it can be higher in other plants, such as in Solanaceous species, where cholesterol is commonly esterified and forms toxic steroidal alkaloids through the GLYCOALKALOID METABOLISM (GAME9) pathway (Cardenas et al., 2016). Second, some of the cholesterol that is made in the shoot is exported to the phloem, where it is translocated to the root (Devarenne et al., 2002; Behmer et al., 2013). Why plants have this kind of selective transport of cholesterol is unknown, but phloem-feeding insects rely on this pathway for the production of the molting hormone, ecdysone, not being able to make cholesterol themselves (Behmer et al., 2013). Altering the movement of cholesterol in plants could have a profound impact on the life 
cycle of phloem-feeding insects and is therefore of great agronomic interest. Third, many of the embryo-lethal mutants in Arabidopsis, such as Fackel and Hydra2, are mutations in the $\Delta^{8,14}$ sterol C-14 reductase, which is in the precursor pathway leading to the production of cholesterol, stigmasterol, and brassicasterol and cannot be chemically compensated by brassinolides alone (Clouse, 2002). Fourth, mutations in later stages of the sterol biosynthetic pathway show mis-localization of PIN2, the auxin transporter, and have defects in cell plate formation (Men et al., 2008).

The change in the localization of PIN2 and the change in endocytosis of other proteins and endocytic labels that accompany these mutations in sterol biosynthesis have been interpreted as evidence for the vesicular movement of sterols from the plasma membrane (PM) to the endosomal compartment via endocytosis (Boutte and Grebe, 2009; Stanislas et al., 2014). Early work (Grebe et al., 2003) supporting this interpretation, using in vivo labeling with the fluorescent polyene antibiotic, filipin, which binds sterols in the PM and also labels endocytic structures, has been reevaluated. Filipin apparently inhibits endocytosis (Boutté et al., 2011) and, when added to root hairs, causes aberrant endocytic structures to form (Oveĉka et al., 2010). This is probably a consequence of the formation of sterolfilipin aggregates in the PM that perturb membrane function (Oveĉka et al., 2010).

Hence, the importance of sterols for the process of endocytosis does not necessarily mean that vesicular endocytosis is the main mechanism for internalization of PM sterols. There are other potential mechanisms for the delivery of sterols to endosomes including transport by cytoplasmic sterol-binding proteins (Iaea et al., 2017) and transport to recycling endosomes via the trans-Golgi network and transport to endosomes via endoplasmic reticulum (ER)-to-endosome membrane contact sites (MCS) (Friedman et al., 2013). If vesicular endocytosis is not the mechanism for internalization of sterol, the other main candidate for sterol (and other lipid) transport from the membrane into the cell in plants is putative ER-PM MCS (Li-Beisson et al., 2013). Although ER-PM MCS or anchor sites have been visualized using persistency mapping (Sparkes et al., 2009) and molecular components of the MCS have been identified (Wang et al., 2014; Levy et al., 2015; PerezSancho et al., 2015), the function of the ER-PM MCS in plants has not been elucidated. Tantalizing evidence that the specialized ER-PM MCS occurring in the plasmodesmata might be involved in sterol biosynthesis or transport comes from the observations that plasmodesmata are enriched in sterols (Grison et al., 2015) and that plasmodesmatal reticulons, ER proteins involved in the tubulation of the ER (Sparkes et al., 2011), can bind to sterol methyl transferase 1 (SMT1) as a partner (Kriechbaumer et al., 2015). The strongest evidence that ER-PM MCS are engaged in sterol transport, however, comes from other systems, such as yeast, where proteins involved in sterol transport, other than oxysterol-binding proteins, have been identified (Gatta et al., 2015).

Therefore, we undertook an investigation into the mechanism of sterol entry into the plant cell to determine whether vesicular or non-vesicular uptake is the primary route of entry of sterols. For this, we use the fluorescent sterols BODIPYcholesterol (BCh) and dehydroergosterol (DHE). Our data are more consistent with a non-vesicular entry of sterols than with an endocytic vesicular uptake of sterols. The immediate target of internalization is a subdomain of the ER, the nuclear envelope (NE), which labels within $5 \mathrm{~min}$ of exposure to fluorescent sterols. Interestingly, there is no accumulation of sterols in other subdomains of the ER, a result which may be expected because even though it is the primary site of sterol biosynthesis within the cell (Hartmann, 2004), there is proportionally less sterol in the ER than in other endomembrane compartments (Moreau et al., 1998b).

\section{MATERIALS AND METHODS}

\section{Plant Materials and Growth Conditions}

The wild-type and transgenic seeds of Arabidopsis thaliana were surface-sterilized with $70 \%$ ethanol and planted on $1 / 2$ strength Murashige and Skoog (Caisson Labs, United States) 1\% agar (Sigma-Aldrich, United States) medium containing vitamins and phosphates, and the $\mathrm{pH}$ was adjusted to between 5.6 and 5.8. Following cold, dark treatment for $48 \mathrm{~h}$ in Petri dishes, the seedlings were grown for 4-5 days at $22^{\circ}$ under continuous white light in a vertical position and treated for analysis. The following homozygous transgenic Arabidopsis lines tagged with fluorescent fusions were used in this study: RFP and GFP targeted to NE (SUN-RFP and SINE2-GFP) (Zhou et al., 2014, 2015), an mCherry-tagged PM marker (NPSN12) (Geldner et al., 2009), an mCherrytagged late endosomal marker (RabF2a-mCherry) (Geldner et al., 2009), an mCherry-tagged trans-Golgi network/early endosomal marker (VTI12-mCherry) (Geldner et al., 2009), and mCherry-HDEL (Cheng et al., 2017). The $\beta$-estradiol-inducible auxilin-2-like (AUX2) Arabidopsis line was from the Friml lab (Adamowski et al., 2018).

\section{BCh, DHE, FM4-64, Filipin, and Nile Red Labeling}

BODIPY-cholesterol (TopFluor ${ }^{\circledR}$, Avanti Polar Lipids, United States) and DHE (Sigma-Aldrich, United States) were prepared as a stock solution with a slight modification from that previously described (Holtta-Vuori et al., 2008). BCh was dissolved in $100 \%$ ethanol to $1.7 \mathrm{mM}$ in the stock solution and DHE at $10 \mathrm{mM}$ in the stock solution and combined with methyl- $\beta$-cyclodextrin $(\mathrm{M} \beta \mathrm{CD})$ (Sigma-Aldrich, United States) at a molar ratio of 1:3 to a final concentration of $10 \mu \mathrm{M} \mathrm{BCh}$ or DHE and $30 \mu \mathrm{M} \mathrm{M} \beta C D$. The probe was either sonicated for $30 \mathrm{~min}$ and vortexed for $15 \mathrm{~min}$ before use or just vortexed for 30 min before use. The styryl dye FM4-64 (Invitrogen, United States) at a final concentration of $4 \mu \mathrm{M}$ was used for dual labeling with BCh. Filipin (Sigma-Aldrich, United States) was made up of a DMSO stock solution (15 $\mathrm{mM})$ and diluted 1:500 in water for treatment. For oil body staining, seedlings were incubated in $2 \mu \mathrm{g} / \mathrm{ml}$ Nile Red (Sigma-Aldrich, United States) solution (made from $2 \mathrm{mg} / \mathrm{ml}$ stock in acetone). 


\section{Confocal Microscopy and Image Analysis}

Confocal laser scanning microscopy imaging was performed on intact Arabidopsis wild-type (Col-0) and transgenic seedlings grown on agar plates. Whole seedlings were stained for the time indicated. Imaging was done on an Olympus FluoView 1000 confocal imaging system equipped with 60X, 1.2 NA water immersion objective. Images and time-lapse videos for analysis were taken using a $20 \mu \mathrm{s} /$ pixel dwell time. BCh $(\lambda$ ex $=497 \mathrm{~nm}$, $\lambda \mathrm{em}=507 \mathrm{~nm}$, Holtta-Vuori et al., 2008) was examined with the $488 \mathrm{~nm}$ line of an argon ion laser, and fluorescence was recorded between 500 and $530 \mathrm{~nm}$. mCherry and RFP fusion proteins were examined with a $543 \mathrm{~nm} \mathrm{He}-\mathrm{Ne}$ laser and fluorescence recorded between 585 and $685 \mathrm{~nm}$. FM4-64 labeling also used the $543 \mathrm{~nm} \mathrm{He}-\mathrm{Ne}$ laser and fluorescence recorded at $640-700 \mathrm{~nm}$ (Bolte et al., 2004). Analysis and post-processing of images were performed with ImageJ (Schneider et al., 2012), FIJI (Schindelin et al., 2012), and Adobe Photoshop ${ }^{\circledR}$ as well as Adobe Illustrator ${ }^{\circledR}$ (CS 6, Adobe Systems, San Jose, CA, United States). The relative intensity of NE was calculated by subtracting the cytoplasmic integrated density per unit area from the NE integrated density per unit area.

\section{Multiphoton Laser Microscopy}

Fluorescent observation of DHE was carried out with multiphoton microscopy. For multiphoton imaging, $10 \mathrm{fs}$ pulses centered at $800 \mathrm{~nm}$ with a bandwidth of $133 \mathrm{~nm}$ were pre-compensated and coupled to a 20X, 1.0 NA objective with $\mathrm{x}-\mathrm{y}$ scanning mirrors. An average power of $25 \mathrm{~mW}$ was used on live samples. Fluorescence signals were separated by a dichroic long-pass mirror at $430 \mathrm{~nm}$. Wavelengths below $430 \mathrm{~nm}$ passed through a BG-39 filter, and wavelengths above $430 \mathrm{~nm}$ passed through a bandpass filter centered at $450 \mathrm{~nm}$ with a bandwidth of $60 \mathrm{~nm}$ to separate the DHE fluorescence and cellular autofluorescence that were subsequently detected with photomultiplier tubes.

\section{RESULTS}

\section{BCh and DHE Track Endogenous Sterols}

BODIPY-cholesterol and DHE $(10 \mu \mathrm{M})$ label a region of native sterol accumulation, the tip of the root hair. Sterols accumulate there (Oveckka et al., 2010), as indicated by their staining with filipin. As shown in Figure 1, filipin, BCh, and DHE label the tip of emerging root hairs, supporting the hypothesis that they are effective tracers of endogenous sterol distribution.

\section{BCh and DHE Label the NE and PM of Elongating Cells of the Root}

Uptake of the DHE into the NE after 30 min was detected with multiphoton microscopy (Figure 2). Colocalization was done in plants expressing SINE2-GFP, an NE protein (Zhou et al., 2014; Figures 2A-H). Line plots of the label across the nucleus produce a double-peaked profile for both DHE and SINE2-GFP, in larger, rounder nuclei (Figures 2A-D), as well as for more typical nuclear profiles, flattened against the PM (Figures $2 \mathbf{E}-\mathbf{H}$ ). Nuclei of plants expressing SUN-RFP also show a two-peaked profile, whereas the cytosolic dye, fluorescein diacetate, shows a broad single peak (Figures 2I-M) because it diffuses from the cytoplasm into the nucleoplasm.

The accumulation of fluorescent sterol in the NE is also seen with BCh. Elongating root cells of plants expressing either SUN-RFP (Zhou et al., 2015) or cytoplasmic mCherry were treated for $30 \mathrm{~min}$ with $\mathrm{BCh}$, and colocalization with the NE was seen (Figures $\mathbf{3 A}-\mathbf{K})$. In cytosolic mCherry-expressing
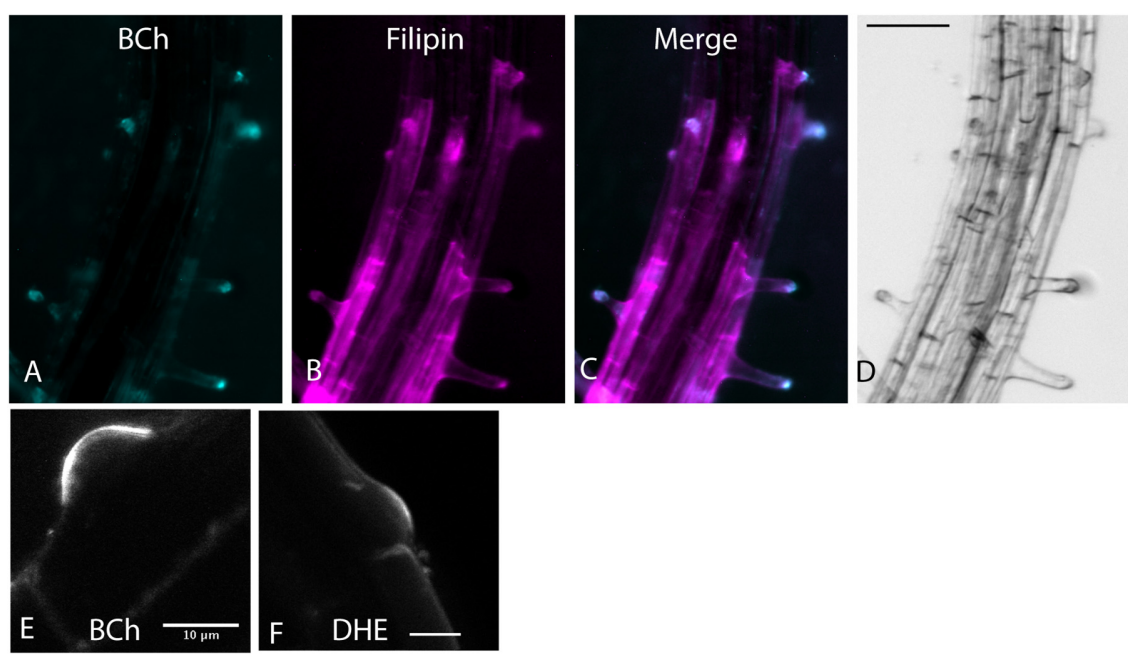

FIGURE 1 | Root hair tip localization of BCh, filipin, and DHE in 5-day-old Arabidopsis seedlings labeled for 60 min with both dyes and $30 \mu \mathrm{M}$ M $\beta$ CD. (A) $10 \mu \mathrm{M}$ BCh localization primarily in the tips of the root hairs. (B) $15 \mu \mathrm{M}$ filipin labeling of root hair tips and along the PM of epidermal cells in the mature regions of the root. (C) Merged image of (A and B). (D) Bright-field image of the root. Scale bar = $100 \mu \mathrm{m}$. (E) Emergent root hair labeled with BCh for 20 min, similar to that shown in the upper part of $\mathbf{A}$. Scale bar $=10 \mu \mathrm{m}$. (F) Emergent root hair at the base of an epidermal cell labeled for $20 \mathrm{~min}$ with $\mathrm{DHE}$. Scale bar $=10 \mu \mathrm{m}$. 

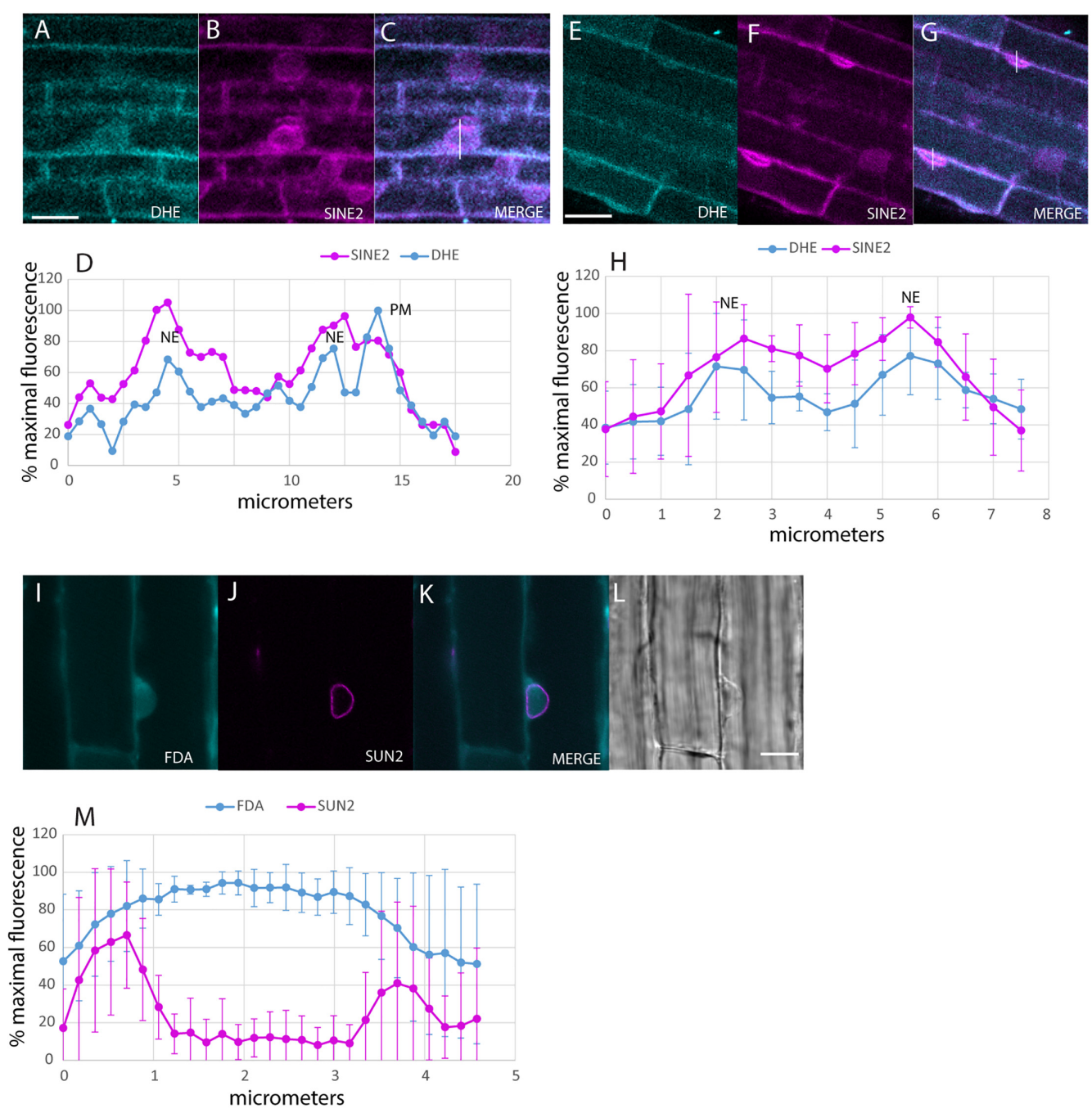

FIGURE 2 | Labeling of the NE with DHE in elongating root cells. (A-C) Seedlings expressing SINE2-GFP were incubated for 30 min in DHE and fluorescence after multiphoton excitation was measured. (A) Emission at 405/20 nm for DHE. (B) Emission at 525/50 nm for SINE2-GFP. (C) Merged image of the DHE signal (cyan) and the SINE2-GFP signal (magenta). Scale bar $=20 \mu \mathrm{m}$. (D) Profile plot of line shown in (C). PM indicates peak from PM. NE indicates peaks from the membranes of the NE. (E-G) Seedlings expressing SINE2-GFP were incubated for $30 \mathrm{~min}$ in DHE as in (A), but the nuclei of these cells lie against the PM. (E) DHE signal. (F) SINE2-GFP signal. (G) Merged image. (H) Averages of profile plots of five similarly sized (smaller than the nucleus in A-D) nuclei show a bimodal distribution of intensity peaking at the NE. The error bars indicate standard deviation. (I-L) Seedlings expressing SUN-RFP were incubated for 30 min in the vital dye fluorescein diacetate to label the cytoplasm. (I) Fluorescein signal. (J) SUN-RFP signal. (K) Merged image. Scale bar $=10 \mu \mathrm{m}$. (L) Bright-field image. (M) Average of five profile plots of nuclei that lie against the PM, including the one shown in (I-L). The error bars indicate standard deviation.

plants, line profiles of the mCherry signal show a single broad peak, indicating that it labels the nucleoplasm as well as the cytoplasm, while line profiles of the BCh label show a two-peaked profile (Figures 3A-D). Line scans across two separate nuclei (Figures 3J,K) reveal that the peaks of SUN-RFP and BCh closely correspond at the NE.

The time course of internalization of the fluorescent sterols and their appearance in the NE is shown in Figures $4 \mathbf{A}, \mathbf{B}$. Labeling of the NE with DHE and BCh can be seen within 5 min. Labeling with both $\mathrm{DHE}$ and $\mathrm{BCh}$ reaches saturation fairly quickly, within $30 \mathrm{~min}$. Some of the label may be in the thin cytoplasmic layer between the vacuole membrane and the NE. To correct for this, the uptake into the NE shown in Figure 4 was determined for BCh in cells expressing SUN-RFP and for DHE in cells expressing SINE2-GFP by outlining a region of the envelope marker expression for quantitation and subtracting the intensity of the sub-PM cytoplasmic label found in nearby regions (Figure 4B).

\section{BCh Label at the PM and Near the Cortical ER}

When plants expressing mCherry-HDEL in the ER were treated with BCh for short periods of time, no colocalization in the cortical ER was seen, and in an attempt to reveal any accumulation in the network, the plants were treated with $\mathrm{BCh}$ 

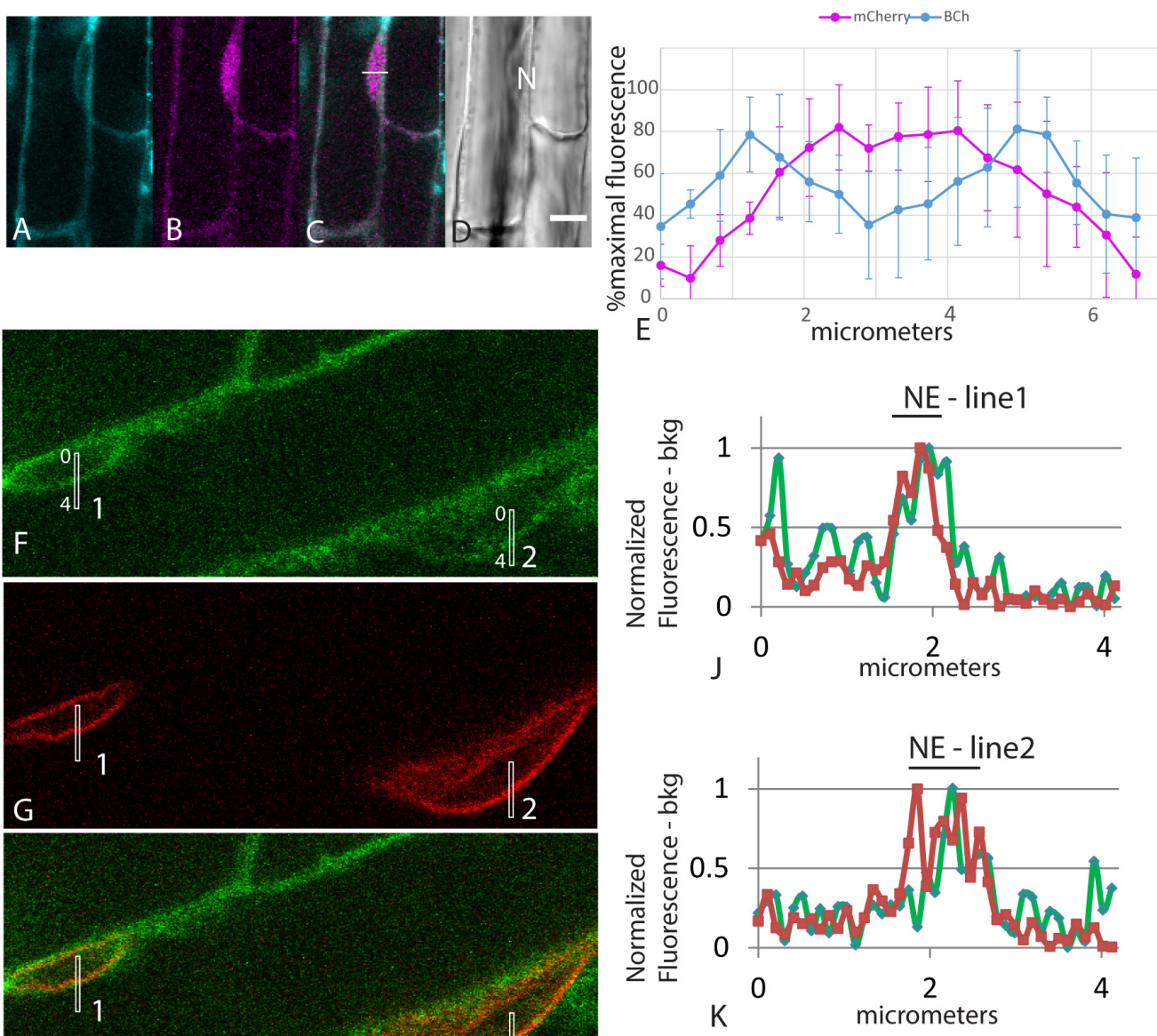

FIGURE 3 | NE label with BCh. (A-E) Comparison of BCh staining after 25 min with expression of cytosolic mCherry. (A) BCh signal. (B) cytoplasmic mCherry signal. (C) Merged image of (A and $\mathbf{B})$, showing the line across which the profile was analyzed. (D) Bright-field image. N, nucleus. Scale bar = 10 m. (E) Average line plot values (plus/minus standard deviation) of four nuclei lying flat against the plasma membrane, as in (A-D). Note that $m$ Cherry has a broad peak and that BCh has two peaks at the edges of the nuclei. (F-K) BCh label after 30 min and colocalization of the label with the NE marker SUN-RFP. (F) BCh labels the PM and the NE of cells in the elongation zone of roots. (G) SUN-RFP expression in the NE of the cells shown in (F). (H) Merged fluorescence micrographs (F,H). Scale bar $=10 \mu \mathrm{m}$. (I) DIC image of the fluorescent region in $\mathbf{( F - H ) . ~ ( J ) ~ F l u o r e s c e n c e ~ i n ~ t h e ~} 4 \mu \mathrm{m}$ line outlined in region 1 of $(\mathbf{F}-\mathbf{H})$ that crosses the NE. (K) Fluorescence in the $4 \mu \mathrm{m}$ line in region 2 of $\mathbf{( F - H ) . ~ I n ~ ( F ~ a n d ~} \mathbf{G})$, the fluorescence intensity in the BCh channel is green, while that in the SUN-RFP channel is red.

for $16 \mathrm{~h}$ (Figure 5). Even at these extended times, although there is a strong label of the NE in cell 1, there is little colocalization between the ER and the BCh in the cortical cytoplasm outside the NE in cell 3, where the cortical ER network is very clear. The label is at or near the plane of the PM, which is emphasized along the boundary between cell 2 and cell 3 (single arrow). A region containing a glancing optical section of the nuclear membrane (double arrows) also shows some BCh label in cell 3. Line profiles of cells from plants expressing NPSN12-mCherry, a PM marker, show colocalization of the BCh (30 min label) with the PM (Figure 6), as well as a label in the NE. The profiles also suggest that there is a cytoplasmic label in the third peak of profile 1 and the first peak of profile 2 .

\section{BCh Does Not Label Early or Late Endosomes}

Very little labeling of endosomes by $\mathrm{BCh}$ was seen when endosomes were visualized with fluorescent fusion protein markers, Rha1 (RabF2a)-mCherry (Geldner et al., 2009) 

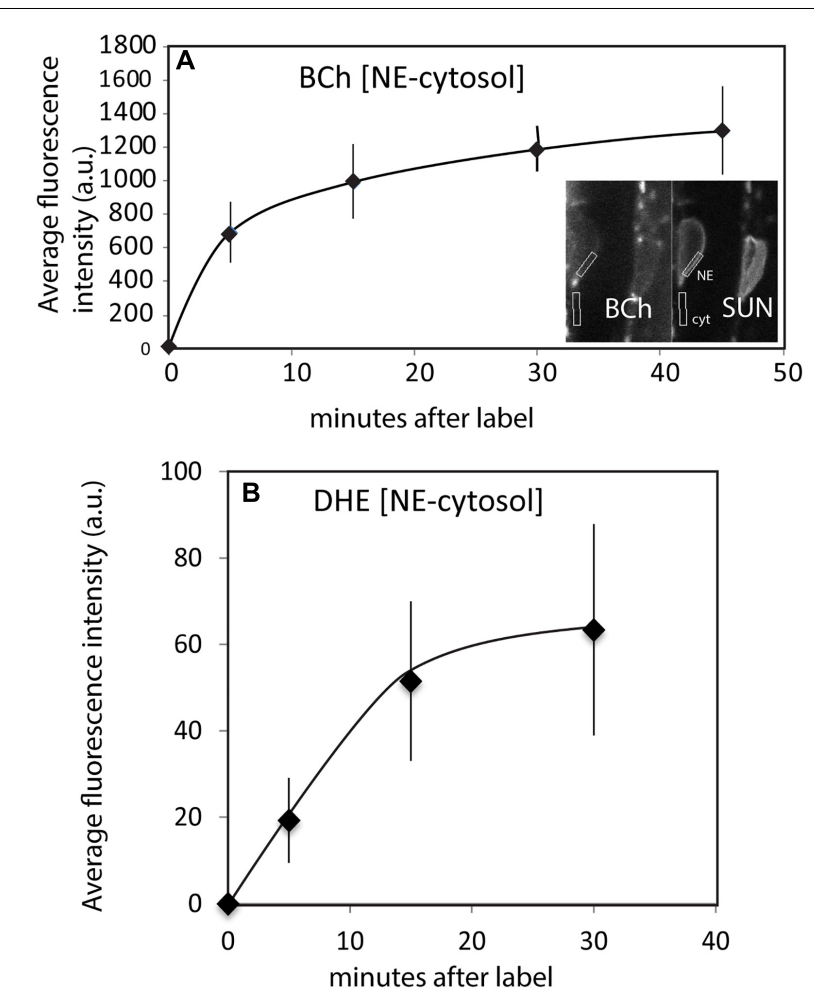

FIGURE 4 | Uptake of BCh and DHE into the NE over time. (A) Average fluorescence intensity of BCh taken over a region containing the NE with an equivalent area of the adjacent cytoplasmic fluorescence subtracted in plants expressing SUN-RFP. Error bars are the standard deviation of five separate samples. Inset shows the identification of the NE and adjacent cytoplasm in the SUN-RFP channel. (B) Average fluorescence intensity of DHE uptake into NE in plants expressing SINE2-GFP. The fluorescence background in the cytoplasmic region has been subtracted. Error bars are the standard deviation of five separate cells.

for the late endosomes and VTI12-mCherry for the early endosomes/trans-Golgi (Geldner et al., 2009; Figure 7). The elongating roots of intact seedlings expressing these fusion protein markers were labeled with BCh for 5-30 min. In all cases, the $\mathrm{BCh}$ does not appreciably label endosomes. However, the $\mathrm{BCh}$ does label the NE and PM (Figures 7B,C).

The absence of a $\mathrm{BCh}$ label in endosomes was confirmed with co-incubation of 5- to 7-day-old seedlings with $4 \mu \mathrm{M}$ FM4-64 and $10 \mu \mathrm{M}$ BCh for $30 \mathrm{~min}$. Epidermal cells of the elongating region of the root were examined in near-tangential optical sections of the outer periclinal cytoplasm (Figure 8). An internal label with BCh showed little colocalization with FM4-64. Several of the regions labeled with BCh were relatively immobile (im, Figure 8C), although there were some moving punctae (mo, Figure 8C). Some of the immobile punctae are refractile in the bright-field image (Figure 8D, rb).

\section{Internalization in Induced AUX2 Overexpression}

To address the possibility that the absence of BCh in endosomes may be a consequence of a low but rapidly transported level of

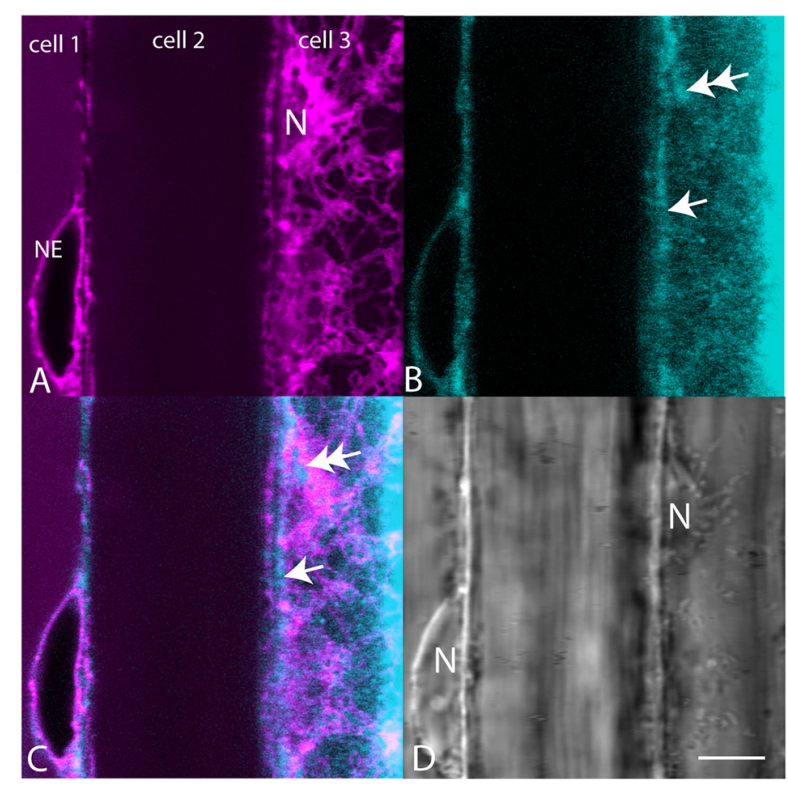

FIGURE 5 | Comparison of mCherry-HDEL label (magenta) of the NE and cortical ER with BCh label (cyan, 16 h). (A) mCherry-HDEL label of the NE in cell 1 and the cortical ER and glancing optical section of the nucleus $(N)$ in cell 3. (B) BCh label of the NE in cell 1 and diffuse label of the cortical region of the cytoplasm in cell 3. Double arrow points to the region on the glancing section of nucleus. Single arrow points to PM. (C) Merged image of (A and B). (D) Bright-field image N, nuclei. The nucleus in cell 3 is in the glancing optical section. Scale bar $=10 \mu \mathrm{m}$.

$\mathrm{BCh}$ in endosomes that is not detected with confocal microscopy, we examined the uptake of BCh after inhibiting endocytosis with the $\beta$-estradiol-inducible expression of AUX2 (Adamowski et al., 2018; Figure 9). After $30 \mathrm{~min}$ of incubation in BCh, the level and pattern of cytoplasmic label in elongating root cells do not change from those seen in wild-type plants (Figure 9A), in wild-type plants preincubated in $\beta$-estradiol (Figure 9B), in AUX2-like plants (Figure 9C), or in AUX2-like plants pretreated with $\beta$-estradiol (Figure 9D). In contrast, uninduced AUX2like plants show normal levels of internalization of FM4-64 (Figure 9E) but show greatly reduced uptake in $\beta$-estradiolinduced AUX2-like plants (Figure 9F). As shown by Adamowski et al. (2018), when the internalization of the FM4-64 is inhibited, it accumulates in pockets and along stretches in the PM (Figure 9F). The quantification of internalization in $\beta$-estradiolinduced and uninduced AUX2-like plants shows significant reduction of internalization of FM4-64 in the induced AUX2 line, but no significant reduction of internalization of $\mathrm{BCh}$ in the induced AUX2 line (Figure 9G).

\section{Nature of the Punctate Label With BCh in Root Cells}

The label with $\mathrm{BCh}$ near the $\mathrm{PM}$ and $\mathrm{NE}$ is somewhat discontinuous (Figure 7) and, when interior, punctate (Figure 8). $\mathrm{BCh}$ can be found in the highly refractile bodies (Figure 8D). Colocalization with Nile Red reveals that these refractile bodies are predominantly oil bodies. Following uptake, the $\mathrm{BCh}$ is 

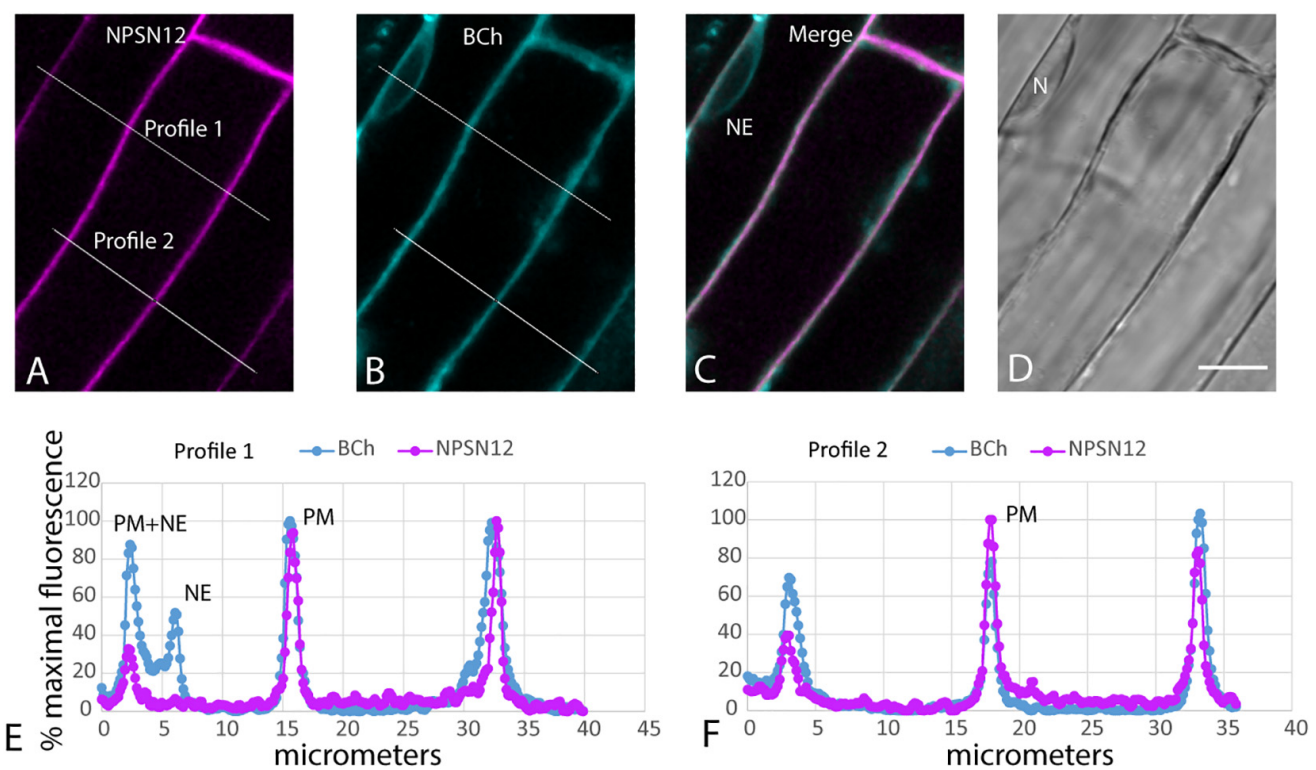

FIGURE 6 | Label with BCh colocalizes with the PM and NE in elongating root cells. Five-day-old seedlings expressing NPSN12-mCherry were treated with BCh for 15 min. (A) PM marker NPSN12-mCherry fluorescence (magenta). (B) BCh signal (cyan). Line profiles in (A and $\mathbf{B})$ are the same, and intensities are plotted in (E and F). (C) Merged image, NE, nuclear envelope. (D) Bright-field image, N, nucleus. Scale bar = $10 \mu \mathrm{m}$. (E) Percentage of maximal fluorescence along line profile 1 in (A) NPSN12-mCherry (magenta) and (B) BCh (cyan). (F) Percentage of maximal fluorescence along line profile 2 in (A) NPSN12-mCherry (magenta) and (B) BCh (cyan). Colocalization at the PM is shown for two different cells at the center peak. NE peaks from (B) are shown as NE in (E).

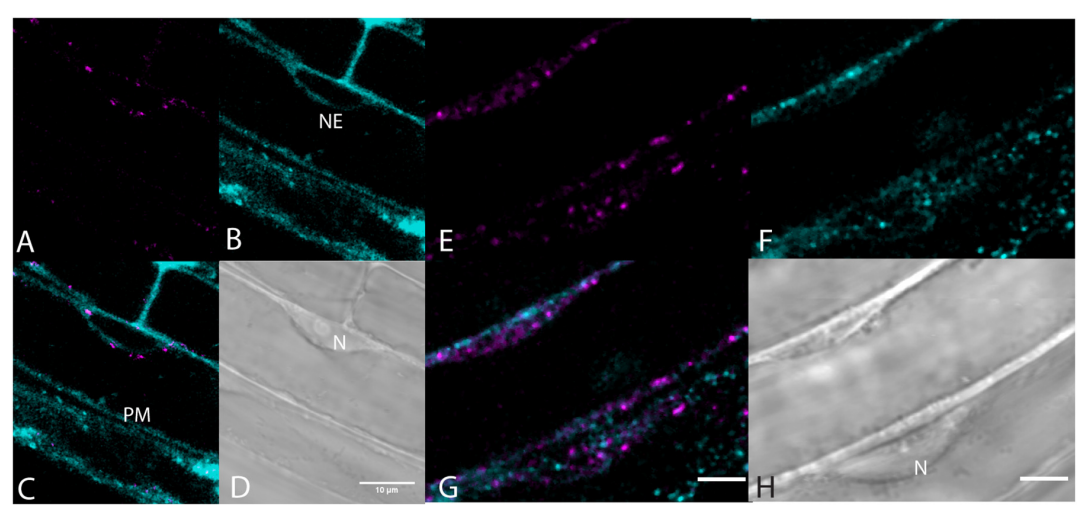

FIGURE 7 | Lack of endosome labels, VTI12-mCherry, and RabF2a-mCherry, with BCh of the elongating root cells. (A-D) Structures labeled after 30 min of incubation in BCh (cyan) in plants expressing the early endosome/trans-Golgi marker, VTI12-mCherry (magenta). (A) Fluorescence of VTI12-mCherry organelles. (B) Fluorescence of BCh labels the NE and the PM. (C) Merged image of (A and B), where magenta endosomes are outside the NE and in the cytoplasm. (D) DIC micrograph showing the nucleus (N). Scale bar = $10 \mu \mathrm{m}$. (E-H) Structures labeled with BCh (cyan) after 30 min in plants expressing RabF2a-mCherry (magenta). (E) Late endosomes labeled with RabF2a-mCherry. (F) BCh (cyan) labels the PM and non-endosomal punctae in the cytosol. (G) Merged image of (E and F) showing magenta endosomes that do not colocalize with the non-endosomal cyan punctae. (H) DIC image showing a glancing optical section of the nucleus (N). Scale bars in $\mathbf{( G )}$ and $\mathbf{( H )}=5 \mu \mathrm{m}$.

probably esterified and BCh esters would accumulate in the lipid body. Long-term incubation in BCh has no effect on growth (Supplementary Figure 1).

\section{DISCUSSION}

Although filipin is not an accurate tracer of the physiological internalization of sterols (Oveĉka et al., 2010), it does accurately label sterol-enriched membranes in fixed tissue and surface sterols in living plants (Boutté et al., 2011). The root hair tip localization of filipin (Figure 1B) is also seen with BCh (Figure 1A). Likewise, when examined with multiphoton microscopy, DHE labels the tip of emerging root hairs, as does BCh (Figures 1A,E,F). This indicates that a modified sterol (BCh) and a foreign sterol (DHE) can be used to accurately trace local sterol distribution. DHE is an analog of ergosterol, a fungal sterol that acts as a signal for the plant cell to initiate a defense response 


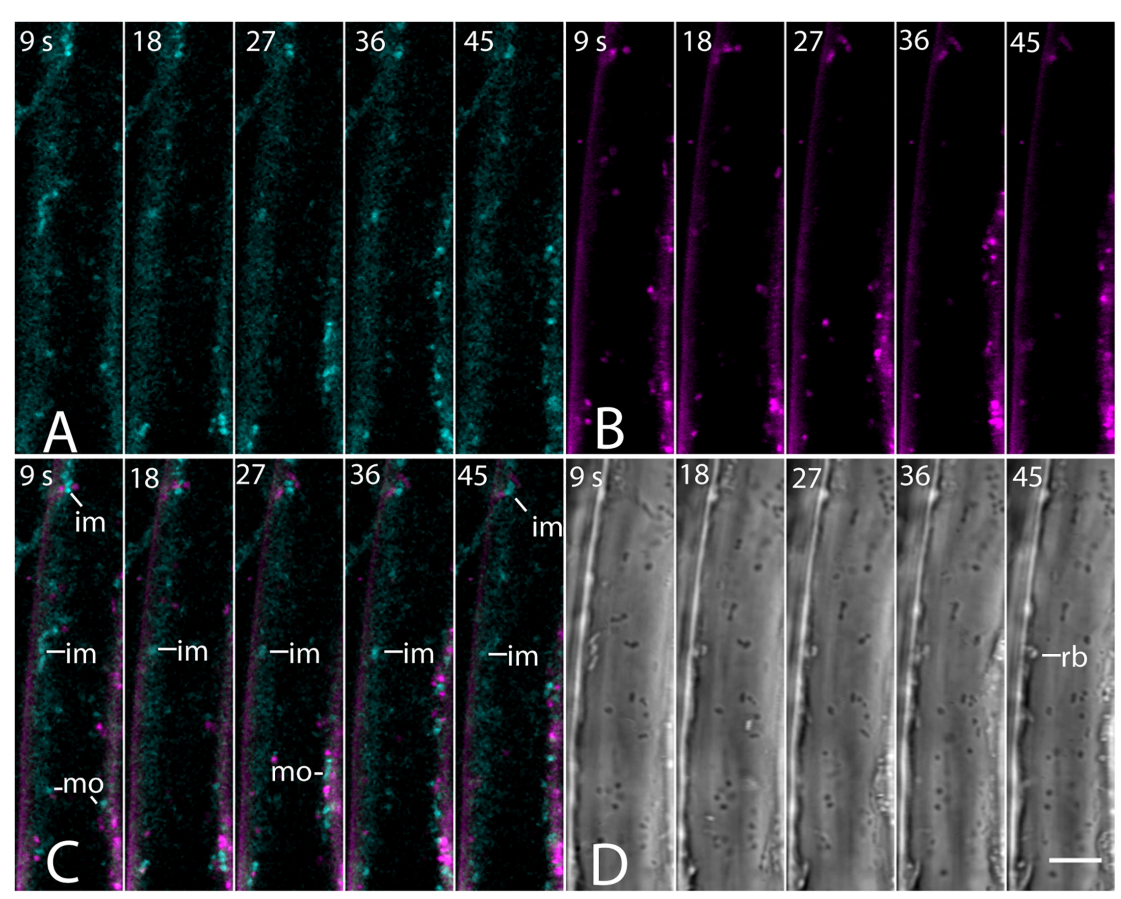

FIGURE 8 | Labeling of elongating root cells with BCh (cyan, A) and FM4-64 (magenta, B) after 30 min of co-incubation. (A) Fluorescent structures associated with the PM and in the cytoplasm. (B) Red fluorescent, mostly motile, endosomes labeled with FM 4-64 after 30 min. (C) Merged image of (A and B) - punctate structures do not colocalize. BCh labels immobile (im) and motile (mo) structures. (D) Bright-field image. rb, refractile body. Images taken at $9 \mathrm{~s}$ intervals. Scale bar $=5 \mu \mathrm{m}$.

(Tugizimana et al., 2014), so long-term (>1 h) treatments are not shown. In the presence of $10 \mu \mathrm{M}$ BCh, on the other hand, plant growth is normal (Supplementary Figure 1).

As shown in Figures 2-4, BCh and DHE both quickly label the NE. The absence of both sterol probes in the nucleoplasm (Figures 2, 3) indicates that they do not simply diffuse through the cytoplasm and into the nucleus as occurs with fluorescein generated from fluorescein diacetate (Figures 2I-M) or cytoplasmic mCherry (Figures 3A-E). An initial (5-10 min) label does not occur in motile, streaming punctae but is found at the PM and the NE. Uptake into the NE is fast. The data do not exclude diffuse uptake into the cytoplasm, but when the level of cytoplasmic fluorescence is subtracted from NE fluorescence (Figure 4), the NE label appears higher than the cytoplasmic label and saturates over time. Furthermore, when a line plot of labeling intensity by $\mathrm{BCh}$ is compared with a marker for the $\mathrm{NE}$, there is little label in the cytoplasmic region outside the region marked by the SUN-RFP, NE signal (Figures 3F-K).

In order to label cells with sterols, they had to be in the presence of the delivery agent, M $\beta C D$. Although M $\beta C D$ has not been used previously in sterol uptake experiments in plants to our knowledge, it has been used to deplete sterols from plant membranes (Roche et al., 2008; Li et al., 2011). M 3 CD apparently dimerizes when it binds sterols (Lopez et al., 2013) and can either extract or supply sterols to a membrane.

The appearance of sterols in the NE is not so surprising when one considers that the concentration of cholesterol (Kemp and Mercer, 1968) and other sterols (Philipp et al., 1976) in the NE is higher than that in the ER in general. There are hypotheses for the function of the accumulation of sterols and other lipids in the $\mathrm{NE}$ in, for instance, yeast whereby their presence is postulated to be a storage mechanism for lipid during the elaboration and growth of the membrane that occurs during cytokinesis (Byrne, 2012; Witkin et al., 2012).

The rest of the ER does not accumulate sterols during their synthesis (Moreau et al., 1998a). The absence of sterols in the ER during their biosynthesis indicates that there is rapid transport out of the ER. This is consistent with the observations in Figure 5, showing that the ER does not accumulate BCh. The label that is in focus in the en face view of the cell cortex, which reveals the ER network, does not form a pattern (Figure 5B) but does label the $\mathrm{NE}$ in the cross section, cell 1 (Figure 5B), that is also labeled with the ER marker (Figure 5A) and in the glancing optical section (Figure 5B, double arrow). This non-patterned, but sometimes punctate, BCh label is mostly in the PM (Figure 6), although there is some label as well in regions of the cytoplasm (Figure 6E, third peak, and Figure 6F, first peak).

The label seen after 30 min of incubation in BCh does not colocalize with early or late endosomes, as assessed by markers for these organelles (Figures 7, 8). Although BCh does label some motile and immotile punctae at this time (Figures 7F, 8A), this label does not colocalize with an early endosome marker (Figures 7A-D) or a late endosome marker (Figures 7E-G). The continuous incubation of the cells with $\mathrm{BCh}$ over this time frame should provide a constant flow of membrane into both early and late endosomes were the $\mathrm{BCh}$ taken up endocytotically. Absence 

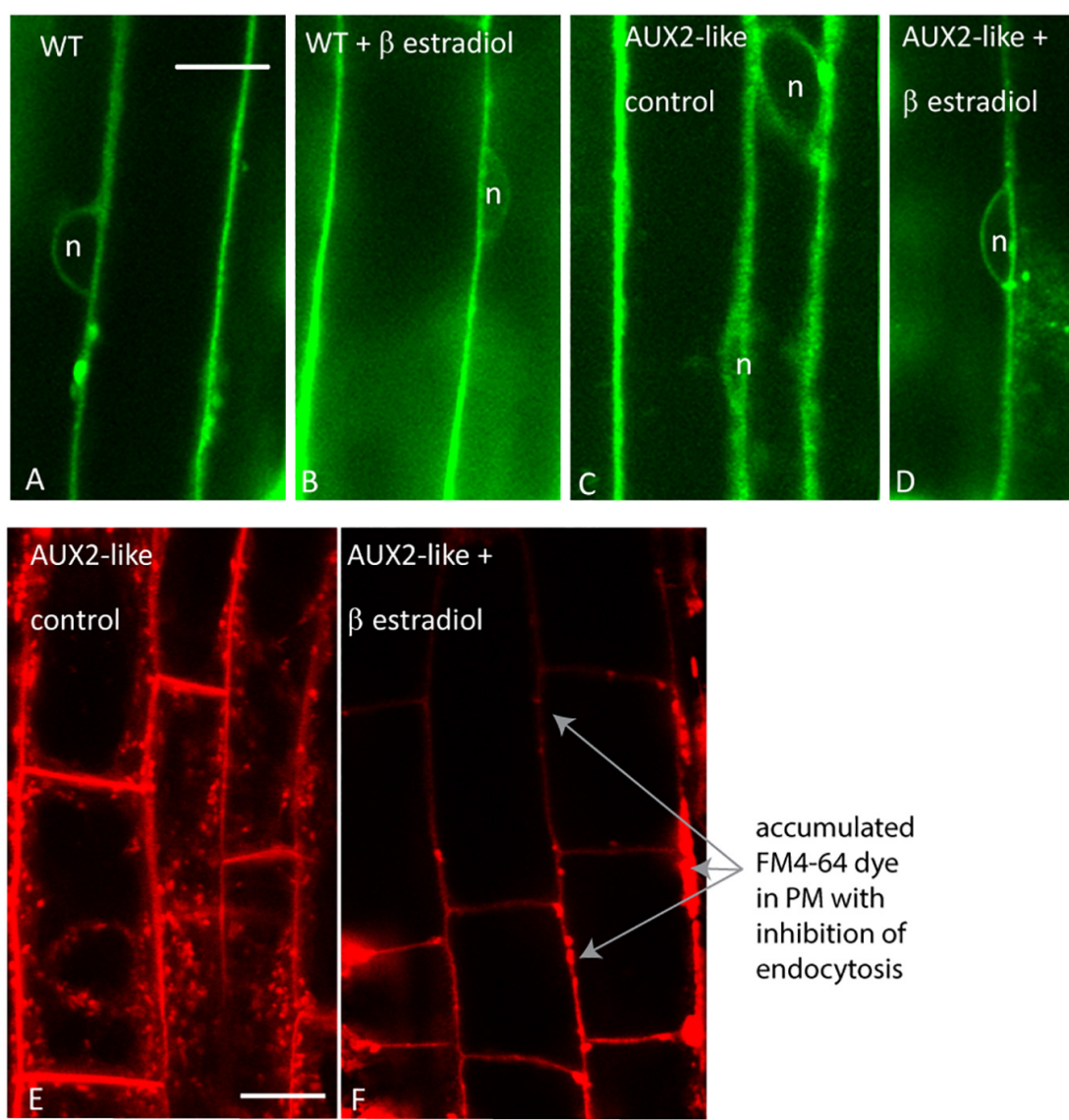

accumulated

FM4-64 dye

in PM with

inhibition of

endocytosis

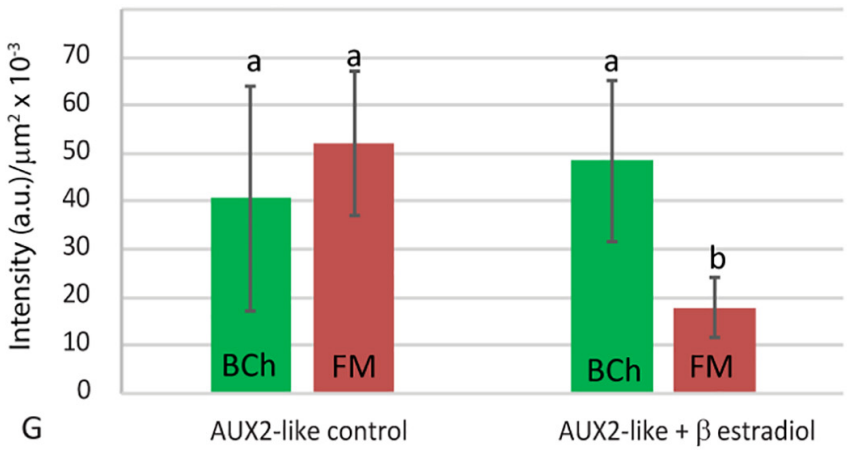

FIGURE 9 | Analysis of uptake in AUX2 expression lines. Confocal micrographs of elongating root cells following (A-D) 30 min labeling with BCh in (A) the wild-type (WT) Arabidopsis line, (B) WT line pretreated with $9 \mathrm{mM} \beta$-estradiol, (C) the AUX2 line, and (D) the AUX2 line pretreated with $9 \mathrm{mM} \beta$-estradiol; (E-F) 30 min labeling with FM4-64 in (E) the AUX2 line and (F) the AUX2 line pretreated with $9 \mathrm{mM} \beta$-estradiol. (G) Quantitation of label intensity per square micrometer of cytoplasm in four to five different cells in three different plants. (b) column values $<0.05 p$ compared to (a) column values, $\mathrm{n}$, nucleus. Scale bars $=10 \mu \mathrm{m}$.

of BCh in the endocytic pathway was confirmed by the absence of colocalization with FM4-64 (Figure 8), a marker of membranebound endocytosis (Griffing, 2008). The presence of BCh would be expected were there endocytosis of sterols through a common, clathrin-mediated endocytic pathway.

However, if the endocytic delivery pathway were extremely transient, as is postulated for the delivery of sterols out of the ER upon their synthesis in the ER, then inhibiting endocytosis should rule it out. This was addressed by following the initial uptake of BCh in plants where endocytosis was inhibited with the induced overexpression of AUX2 protein (Adamowski et al., 2018). With the induction of overexpression of AUX2 protein with $\beta$-estradiol, endocytosis of FM4-64 is inhibited (Figures 9EG). The aberrant accumulation of FM4-64 in regions of the PM under these conditions (Figure 9F) repeats the earlier work of Adamowski et al. (2018). The remaining level of the FM464 label quantified in Figure 8G may be the consequence of the accumulation of FM4-64 in PM domains that appear with 


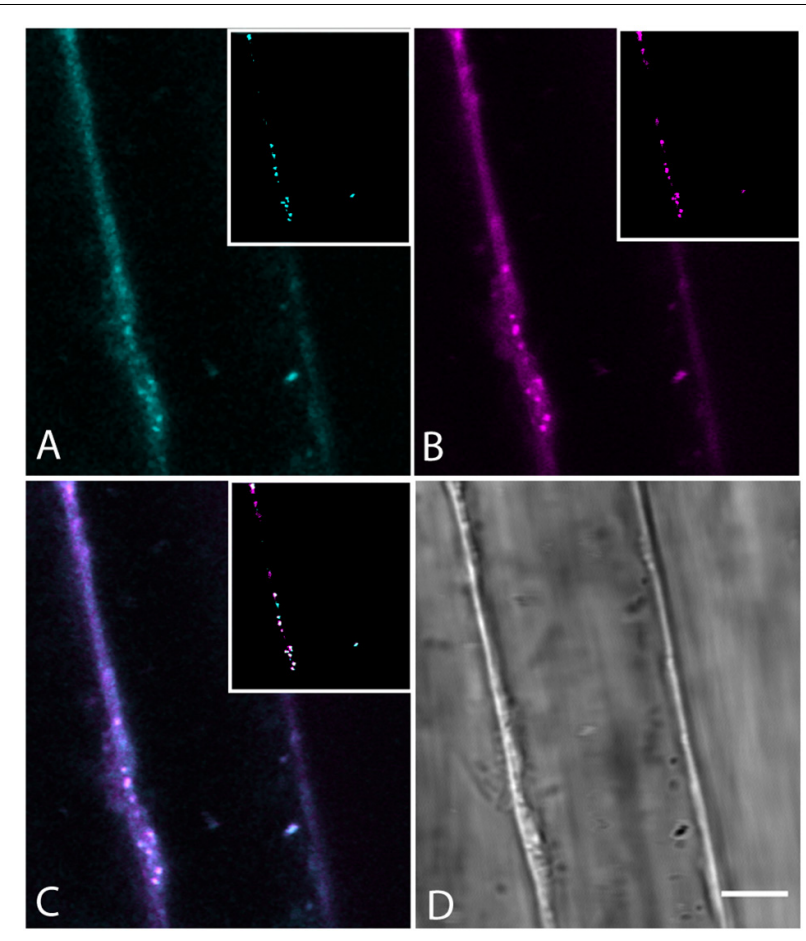

FIGURE 10 | Colocalization of BCh in Nile Red-labeled oil bodies in elongating root cells labeled for 30 min with both probes. (A) Fluorescence of $\mathrm{BCh}$ at the PM and in the cytoplasm. The inset is a cyan binary mask of the brightest organelles in the image (>128 of 256 gray levels) in the region of the cell shown. (B) Fluorescence of Nile Red at the PM and cytoplasm. The inset is a magenta binary mask of the brightest organelles in the image ( $>128$ of 256 gray levels) in the region of the cell shown and marks the oil bodies. (C) Merged image of (A and $\mathbf{B}$ ), where the inset is a composite mask of the insets in (A and $\mathbf{B})$, showing that the majority of bright organelles are white, or colocalized. (D) Bright-field micrograph of the cell. Scale bar $=5 \mu \mathrm{m}$.

inhibition of endocytosis. However, the uptake of BCh into the NE is not inhibited (Figures 9A-D,G).

The non-vesicular mode of uptake of sterols can be found in other systems, where it is proposed that the sterols are internalized by specific transporters that are associated with ERPM MCS (Gatta et al., 2015; Lahiri et al., 2015). Of particular interest is the work of Gatta et al. (2015), where it was shown that internalization of sterols in yeast occurred in a genetic background where all seven oxysterol-binding proteins, also thought to be involved in sterol transport, had been deleted. The sterol transport capability of yeast cells seemed to be conferred, at least in part, by StART-like proteins, since mutations in these proteins inhibited the uptake of sterols. In that work, the internalization of DHE and cholesterol was monitored by the internal formation of sterol esters and delivery to oil bodies. The first appearance of sterol internalization was at 10-20 min. Here, we have shown that internalization can be detected within 5 min of exposure to BCh (Figure 4). Intriguingly, some of the StART-like proteins in plants have a transmembrane spanning domain in the middle of the protein (Gatta et al., 2015), which may form a single hairpin structure in the cytoplasmic leaflet of the ER. Plant ER proteins known to reside in the cytoplasmic leaflet of the ER, i.e., the membrane-bending reticulons (Zurek et al., 2011) and the large GTP-binding protein involved in ER fusion and/or bundling, Root Hair Defective 3 (RHD3) (Ueda et al., 2016), have a double-hairpin structure. If these StART-like proteins associate with these other proteins on the cytoplasmic side of the ER and are localized to ER-PM MCS, they are excellent candidates for a sterol transporter in plants. In fact, it has recently been shown that one of the reticulons localized to the outer membrane of the ER in plants, RTN20, changes sterol dynamics (Kriechbaumer et al., 2018).

The compartment of neutral lipids, the oil body, is a wellknown place in plants for the storage of lipids and modified sterols and presumably originate from the ER. Overproduction of sterols produces oil bodies containing higher levels of sterol esters (Gondet et al., 1994). Sterol homeostasis is mediated by phospholipid sterol acyltransferase 1, and plants deficient in this enzyme do not accumulate as much lipid in oil bodies (BouvierNave et al., 2010). BCh colocalization with oil bodies (Figure 10) indicates that oil bodies take up and store the extra sterol. The observation is consistent with the model that oil body sterol esters, as in yeast, arise from the ER and are stored inside the oil bodies (Gatta et al., 2015).

\section{DATA AVAILABILITY STATEMENT}

The original contributions presented in the study are included in the article/Supplementary Material, further inquiries can be directed to the corresponding author.

\section{AUTHOR CONTRIBUTIONS}

LG and KK designed and carried out the experiments. HG and AY carried out the multiphoton microscopy experiments with assistance from LG and KK. LG wrote the manuscript. All authors contributed to the article and approved the submitted version.

\section{FUNDING}

Funding from the Department of Biology, the Texas A\&M University Triad 3 funding program \#02-247034 (LG and AY) and the use of the facilities in the Microscopy and Imaging Center at Texas A\&M University are gratefully acknowledged.

\section{SUPPLEMENTARY MATERIAL}

The Supplementary Material for this article can be found online at: https://www.frontiersin.org/articles/10.3389/fpls.2021. 616631/full\#supplementary-material

Supplementary Figure 1 | Absence of growth effects on seedlings grown in the presence of BCh. (A) Plate of untreated Arabidopsis seedlings. (B) Plate of Arabidopsis seedlings grown in the presence of $10 \mu \mathrm{M}$ BCh. (C) Root length measured over 3, 5, and 7 days after planting (DAP). (D) Hypocotyl length measured over 3, 5, and 7 DAP. diamonds, control; squares, BCh treated. 


\section{REFERENCES}

Adamowski, M., Narasimhan, M., Kania, U., Glanc, M., De Jaeger, G., and Friml, J. (2018). A functional study of AUXILIN-LIKE1 and 2, two putative clathrin uncoating factors in Arabidopsis. Plant Cell 30, 700-716. doi: 10.1105/tpc.17. 00785

Behmer, S. T., Olszewski, N., Sebastiani, J., Palka, S., Sparacino, G., Sciarrno, E., et al. (2013). Plant phloem sterol content: forms, putative functions, and implications for phloem-feeding insects. Front. Plant Sci. 4:370. doi: 10.3389/ fpls.2013.00370

Behrman, E. J., and Gopalan, V. (2005). Cholesterol and plants. J. Chem. Educ. 82, 1791-1793. doi: 10.1021/ed082p1791

Bolte, S., Talbot, C., Boutte, Y., Catrice, O., Read, N. D., and Satiat-Jeunemaitre, B. (2004). FM-dyes as experimental probes for dissecting vesicle trafficking in living plant cells. J. Microsc. 214, 159-173. doi: 10.1111/j.0022-2720.2004. 01348.x

Boutte, Y., and Grebe, M. (2009). Cellular processes relying on sterol function in plants. Curr. Opin. Plant Biol. 12, 705-713. doi: 10.1016/j.pbi.2009.09.013

Boutté, Y., Men, S., and Grebe, M. (2011). Fluorescent in situ visualization of sterols in Arabidopsis roots. Nat. Protoc. 6, 446-456. doi: 10.1038/nprot.2011.323

Bouvier-Nave, P., Berna, A., Noiriel, A., Compagnon, V., Carlsson, A. S., Banas, A., et al. (2010). Involvement of the phospholipid sterol acyltransferase1 in plant sterol homeostasis and leaf senescence. Plant Physiol. 152, 107-119. doi: 10.1104/pp.109.145672

Byrne, R. D. (2012). The nuclear membrane as a lipid 'sink'-linking cell cycle progression to lipid synthesis. J. Chem. Biol. 5, 141-142. doi: 10.1007/s12154012-0082-1

Cardenas, P. D., Sonawane, P. D., Pollier, J., Vanden Bossche, R., Dewangan, V., Weithorn, E., et al. (2016). GAME9 regulates the biosynthesis of steroidal alkaloids and upstream isoprenoids in the plant mevalonate pathway. Nat. Commun. 7:10654.

Cheng, X., Lang, I., Adeniji, O. S., and Griffing, L. (2017). Plasmolysisdeplasmolysis causes changes in endoplasmic reticulum form, movement, flow, and cytoskeletal association. J. Exp. Bot. 68, 4075-4087. doi: 10.1093/jxb/erx243

Clouse, S. D. (2002). Arabidopsis mutants reveal multiple roles for sterols in plant development. Plant Cell 14, 1995-2000. doi: 10.1105/tpc.140930

Devarenne, T. P., Ghosh, A., and Chappell, J. (2002). Regulation of squalene synthase, a key enzyme of sterol biosynthesis, in tobacco. Plant Physiol. 129, 1095-1106. doi: 10.1104/pp.001438

Friedman, J. R., Dibenedetto, J. R., West, M., Rowland, A. A., and Voeltz, G. K. (2013). Endoplasmic reticulum-endosome contact increases as endosomes traffic and mature. Mol. Biol. Cell 24, 1030-1040. doi: 10.1091/mbc.e12-100733

Gatta, A. T., Wong, L. H., Sere, Y. Y., Calderon-Norena, D. M., Cockcroft, S., Menon, A. K., et al. (2015). A new family of StART domain proteins at membrane contact sites has a role in ER-PM sterol transport. eLife 4:e07253. doi: $10.7554 /$ eLife. 07253

Geldner, N., Denervaud-Tendon, V., Hyman, D. L., Mayer, U., Stierhof, Y. D., and Chory, J. (2009). Rapid, combinatorial analysis of membrane compartments in intact plants with a multicolor marker set. Plant J. 59, 169-178. doi: 10.1111/j. 1365-313x.2009.03851.x

Gondet, L., Bronner, R., and Benveniste, P. (1994). Regulation of sterol content in membranes by subcellular compartmentation of Steryl-esters accumulating in a sterol-overproducing Tobacco mutant. Plant Physiol. 105, 509-518. doi: 10.1104/pp.105.2.509

Grebe, M., Xu, J., Mobius, W., Ueda, T., Nakano, A., Geuze, H. J., et al. (2003) Arabidopsis sterol endocytosis involves actin-mediated trafficking via ARA6positive early endosomes. Curr. Biol. 13, 1378-1387. doi: 10.1016/s09609822(03)00538-4

Griffing, L. R. (2008). FRET analysis of transmembrane flipping of FM4-64 in plant cells: Is FM4-64 a robust marker for endocytosis? J. Microsc. 231, 291-298. doi: 10.1111/j.1365-2818.2008.02042.x

Grison, M. S., Brocard, L., Fouillen, L., Nicolas, W., Wewer, V., Dormann, P., et al. (2015). Specific membrane lipid composition is important for plasmodesmata function in Arabidopsis. Plant Cell 27, 1228-1250. doi: 10.1105/tpc.114.135731

Hartmann, M. A. (2004). "5 Sterol metabolism and functions in higher plants," in Lipid Metabolism and Membrane Biogenesis, ed. G. Daum (Berlin: SpringerVerlag), 183-211. doi: 10.1007/978-3-540-40999-1_6
Holtta-Vuori, M., Uronen, R. L., Repakova, J., Salonen, E., Vattulainen, I., Panula, P., et al. (2008). BODIPY-cholesterol: a new tool to visualize sterol trafficking in living cells and organisms. Traffic 9, 1839-1849. doi: 10.1111/j.1600-0854.2008. 00801.x

Iaea, D. B., Mao, S., Lund, F. W., and Maxfield, F. R. (2017). Role of STARD4 in sterol transport between the endocytic recycling compartment and the plasma membrane. Mol. Biol. Cell 28, 1111-1122. doi: 10.1091/mbc.e16-07-0499

Kemp, R. J., and Mercer, E. I. (1968). Studies on the sterols and sterol esters of the intracellular organelles of maize shoots. Biochem. J. 110, 119-125. doi: 10.1042/bj1100119

Kriechbaumer, V., Botchway, S. W., Slade, S. E., Knox, K., Frigerio, L., Oparka, K., et al. (2015). Reticulomics: protein-protein interaction studies with two plasmodesmata-localized reticulon family proteins identify binding partners enriched at plasmodesmata, endoplasmic reticulum, and the plasma membrane. Plant Physiol. 169, 1933-1945.

Kriechbaumer, V., Maneta-Peyret, L., Fouillen, L., Botchway, S. W., Upson, J., Hughes, L., et al. (2018). The odd one out: Arabidopsis reticulon 20 does not bend ER membranes but has a role in lipid regulation. Sci. Rep. 8:2310.

Lahiri, S., Toulmay, A., and Prinz, W. A. (2015). Membrane contact sites, gateways for lipid homeostasis. Curr. Opin. Cell Biol. 33, 82-87. doi: 10.1016/j.ceb.2014. 12.004

Levy, A., Zheng, J. Y., and Lazarowitz, S. G. (2015). Synaptotagmin SYTA forms ER-plasma membrane junctions that are recruited to plasmodesmata for plant virus movement. Curr. Biol. 25, 2018-2025. doi: 10.1016/j.cub.2015.06.015

Li, X., Wang, X., Yang, Y., Li, R., He, Q., Fang, X., et al. (2011). Single-molecule analysis of PIP2;1 dynamics and partitioning reveals multiple modes of Arabidopsis plasma membrane aquaporin regulation. Plant Cell 23, 3780-3797.

Li-Beisson, Y., Shorrosh, B., Beisson, F., Andersson, M. X., Arondel, V., Bates, P. D., et al. (2013). Acyl-lipid metabolism. Arabidopsis Book 11:e0161.

Lopez, C. A., De Vries, A. H., and Marrink, S. J. (2013). Computational microscopy of cyclodextrin mediated cholesterol extraction from lipid model membranes. Sci. Rep. 3:2071.

Men, S., Boutte, Y., Ikeda, Y., Li, X., Palme, K., Stierhof, Y. D., et al. (2008). Steroldependent endocytosis mediates post-cytokinetic acquisition of PIN2 auxin efflux carrier polarity. Nat. Cell Biol. 10, 237-244. doi: 10.1038/ncb1686

Moreau, P., Bessoule, J. J., Mongrand, S., Testet, E., Vincent, P., and Cassagne, C. (1998a). Lipid trafficking in plant cells. Prog. Lipid Res. 37, 371-391. doi: 10.1016/s0163-7827(98)00016-2

Moreau, P., Hartmann, M. A., Perret, A. M., Sturbois-Balcerzak, B., and Cassagne, C. (1998b). Transport of sterols to the plasma membrane of leek seedlings. Plant Physiol. 117, 931-937. doi: 10.1104/pp.117.3.931

Oveĉka, M., Berson, T., Beck, M., Derksen, J., Samaj, J., Baluska, F., et al. (2010). Structural sterols are involved in both the initiation and tip growth of root hairs in Arabidopsis thaliana. Plant Cell 22, 2999-3019. doi: 10.1105/tpc.109. 069880

Perez-Sancho, J., Vanneste, S., Lee, E., Mcfarlane, H. E., Esteban Del Valle, A., Valpuesta, V., et al. (2015). The Arabidopsis synaptotagminl is enriched in endoplasmic reticulum-plasma membrane contact sites and confers cellular resistance to mechanical stresses. Plant Physiol. 168, 132-143. doi: 10.1104/pp. 15.00260

Philipp, E. I., Franke, W. W., Keenan, T. W., Stadler, J., and Jarasch, E. D. (1976). Characterization of nuclear membranes and endoplasmic reticulum isolated from plant tissue. J. Cell Biol. 68, 11-29. doi: 10.1083/jcb.68.1.11

Roche, Y., Gerbeau-Pissot, P., Buhot, B., Thomas, D., Bonneau, L., Gresti, J., et al. (2008). Depletion of phytosterols from the plant plasma membrane provides evidence for disruption of lipid rafts. FASEB J. 22, 3980-3991. doi: 10.1096/fj. 08-111070

Schindelin, J., Arganda-Carreras, I., Frise, E., Kaynig, V., Longair, M., Pietzsch, T., et al. (2012). Fiji: an open-source platform for biological-image analysis. Nat. Methods 9, 676-682. doi: 10.1038/nmeth.2019

Schneider, C. A., Rasband, W. S., and Eliceiri, K. W. (2012). NIH Image to ImageJ: 25 years of image analysis. Nat. Methods 9, 671-675. doi: 10.1038/nmeth.2089

Sonawane, P. D., Pollier, J., Panda, S., Szymanski, J., Massalha, H., Yona, M., et al. (2016). Plant cholesterol biosynthetic pathway overlaps with phytosterol metabolism. Nat. Plants 3:16205.

Sparkes, I., Hawes, C., and Frigerio, L. (2011). FrontiERs: movers and shapers of the higher plant cortical endoplasmic reticulum. Curr. Opin. Plant Biol. 14, 658-665. doi: 10.1016/j.pbi.2011.07.006 
Sparkes, I., Runions, J., Hawes, C., and Griffing, L. (2009). Movement and remodeling of the endoplasmic reticulum in nondividing cells of tobacco leaves. Plant Cell 21, 3937-3949. doi: 10.1105/tpc.109.072249

Stanislas, T., Grebe, M., and Boutte, Y. (2014). "Sterol dynamics during endocytic trafficking in Arabidopsis," in Plant Endosomes. Methods in Molecular Biology (Methods and Protocols), ed. M. Otegui (New York, NY: Humana Press), 13-29. doi: 10.1007/978-1-4939-1420-3_2

Tugizimana, F., Steenkamp, P. A., Piater, L. A., and Dubery, I. A. (2014). Multi-platform metabolomic analyses of ergosterol-induced dynamic changes in Nicotiana tabacum cells. PLoS One 9:e87846. doi: 10.1371/journal.pone. 0087846

Ueda, H., Yokota, E., Kuwata, K., Kutsuna, N., Mano, S., Shimada, T., et al. (2016). Phosphorylation of the C terminus of RHD3 Has a critical role in Homotypic ER membrane fusion in Arabidopsis. Plant Physiol. 170, 867-880. doi: 10.1104/pp.15.01172

Wang, P., Hawkins, T. J., Richardson, C., Cummins, I., Deeks, M. J., Sparkes, I., et al. (2014). The plant cytoskeleton, NET3C, and VAP27 mediate the link between the plasma membrane and endoplasmic reticulum. Curr. Biol. 24, 1397-1405. doi: 10.1016/j.cub.2014.05.003

Witkin, K. L., Chong, Y., Shao, S., Webster, M. T., Lahiri, S., Walters, A. D., et al. (2012). The budding yeast nuclear envelope adjacent to the nucleolus serves as a membrane sink during mitotic delay. Curr. Biol. 22, 1128-1133. doi: 10.1016/j.cub.2012.04.022
Zhou, X., Graumann, K., Wirthmueller, L., Jones, J. D., and Meier, I. (2014) Identification of unique SUN-interacting nuclear envelope proteins with diverse functions in plants. J. Cell Biol. 205, 677-692. doi: 10.1083/jcb. 201401138

Zhou, X., Groves, N. R., and Meier, I. (2015). Plant nuclear shape is independently determined by the SUN-WIP-WIT2-myosin XI-i complex and CRWN1. Nucleus 6, 144-153. doi: 10.1080/19491034.2014. 1003512

Zurek, N., Sparks, L., and Voeltz, G. (2011). Reticulon short hairpin transmembrane domains are used to shape ER tubules. Traffic 12, 28-41. doi: 10.1111/j.1600-0854.2010.01134.x

Conflict of Interest: The authors declare that the research was conducted in the absence of any commercial or financial relationships that could be construed as a potential conflict of interest.

Copyright (c) 2021 Kumar, Gibbs, Yeh and Griffing. This is an open-access article distributed under the terms of the Creative Commons Attribution License (CC BY). The use, distribution or reproduction in other forums is permitted, provided the original author(s) and the copyright owner(s) are credited and that the original publication in this journal is cited, in accordance with accepted academic practice. No use, distribution or reproduction is permitted which does not comply with these terms. 